\title{
I dentificación de la Propiedad y Dinámica de la Oferta Educativa Particular Subvencionada de la Región Metropolitana
}

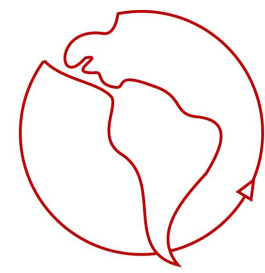

Francisca Corbalán

Psicóloga, Universidad de Chile fcorbalanp@gmail.com

Sebastián Ligüeño

Psicólogo, Universidad de Chile sebastianligueno@yahoo.com

J uan González

Psicólogo, Universidad de Chile juangl@uchile.cl

Resumen

El presente artículo da cuenta de un primer intento de descripción de la configuración de la oferta particular subvencionada en la Región Metropolitana. Para ello, se recogió información del MINEDUC y de las bases de datos del Diario Oficial, lo que permitió conocer la identidad de la constitución de las personas naturales y jurídicas que participan en este sector privado financiado por el Estado. Sobre la base de un análisis de material cuantitativo y cualitativo se propone una nueva categorización de los sostenedores como actores del mercado educativo. Por último, se discuten algunas implicancias de los resultados de la investigación.

\section{Abstract}

This article describes the configuration of the particular educational offer subsidized in the metropolitan area. To this end, information was collected from MINEDUC and databases of the Chilean "Diario Oficial", allowing the identity of the constitution of natural and legal persons involved in the private sector financed by the state. Based on an analysis of qualitative and quantitative material proposes a new categorization of actors as supporters of the education market. Finally, we discuss some implications of research results.

Palabras Clave: Educación, Sostenedor, Educación Particular Subvencionada, Cuasimercado educativo Keywords: Education, Supporters, Private education, quasi educational market

\section{Introducción}

El Sistema Educativo chileno sufrió grandes transformaciones a comienzos de la década de 1980, en el marco de la dictadura militar. Estas modificaciones apuntaron a masificar la escuela básica y media introduciendo mecanismos de mercado. Esto implicó, por consiguiente, instalar la lógica de la competencia en educación y garantizar la existencia de una oferta educativa privada que pudiese absorber la población desescolarizada y/o gestionar algunos establecimientos estatales. La privatización de la provisión educativa pública es la base de la estrategia educativa del gobierno de aquel entonces. Se delega la responsabilidad educativa que históricamente 
el Estado chileno había asumido a los llamados "sostenedores educacionales", personas naturales o jurídicas que son propietarias de establecimientos de enseñanza obligatoria (Constitución Política de 1980) ${ }^{1}$.

Como se aprecia en diversos documentos pro- mercado en Educación (por ejemplo, Jofré 1988), el cambio de sistema educativo no se debió a una discusión pública ni a un diagnóstico que aludiera a sus logros, encontrándose una carencia de evidencia que sustentara la decisión tomada. Por tanto, estas reformas implicaban más bien una concordancia con las políticas privatizadoras neoliberales llevadas a cabo por el gobierno (Nef, 1999-2000).

La inspiración ideológica en la cual se sustentaron las reformas emprendidas en Educación se encuentra en la estrategia general de organismos internacionales, gobiernos y teóricos ad hoc respecto a la crisis de acumulación de la ganancia, enfrentándola mediante el viraje de un Estado de Bienestar a un Estado subsidiario privatizador y controlador. Un ejemplo de esto lo representa el puntal de la Escuela de Chicago, Milton Friedman (mentor ideológico de los economistas chilenos emigrados a partir de la década de los '60, los Chicago Boys), que propone un sistema educativo que se encuentre dirigido por las fuerzas del mercado, en donde el Estado otorgue cupones (vouchers) a los padres para que éstos pudieran escoger el mejor colegio para sus hijos, para lo cual podrían ser elegibles establecimientos privados (Friedman, 1956). El argumento central de Friedman es que los proveedores privados podrían otorgar una educación de la misma calidad a un menor precio, gracias a la competencia por captar estudiantes.

Durante los `90, en los gobiernos de la Concertación ${ }^{2}$, se le hicieron modificaciones al marco legal para el sistema de sostenedores. En 1993, mientras Jorge Arrate era Ministro de Educación, se incorpora la figura del Financimiento Compartido (en adelante FICOM) o copago de la familia en la educación subvencionada por el Estado, que da la posibilidad a todo establecimiento subvencionado de cobrar a la padres un pago adicional a la subvención, la que se reduce de manera proporcional según el tramo que cancelan las familias. Estos cambios se introducen a través de reformas tributarias que incluyen la Ley de Donaciones de las Empresas con Fines Educacionales, así como modificaciones del Decreto con Fuerza de Ley 2 de 1992. Posteriormente, el DFL 2 de 1998 concentra las disposiciones completas del sistema, con las incorporaciones posteriores, que rigen en general hoy para las subvenciones.

Cabe hacer notar que el modelo de Educación basada en mecanismos de mercado se ha perfeccionado durante este período, no obstante ciertos elementos de reversión, que no han tocado los pilares fundamentales de lo que podría llamarse la "Escuela Chilena Mercantilizada". Buen ejemplo de ello es la recientemente promulgada Ley de Subvención Preferencial (Ley Nr. 20.248, 2 de febrero de 2008), que otorga un monto adicional de subvención a los establecimientos de enseñaza básica según la cantidad y concentración de niños vulnerables socioeconómicamente, puesto que estos "son más caros de educar" (Mineduc, 2009).

A más de 25 años de transformación del sistema, la evidencia indica que, cuando se evalúa el impacto de estas políticas, se muestra que no sólo no han mejorado la calidad de la educación, sino que también han incidido profundamente en una segregación del sistema educacional, en términos de la composición de estudiantes por nivel socioeconómico (Carnoy y Mc Ewan, 2003; OCDE, 2004; Redondo et Al 2004; Cornejo, 2006; Bellei, 2007). Un reciente estudio describe cómo en los últimos 15 años 
esta segregación ha ido en aumento, lo que se explica en parte por la alta segregación residencial, y en parte, por el efecto FICOM (Valenzuela, Bellei y de los Ríos, 2008).

De forma complementaria, una hipótesis subyacente es que las escuelas privadas subvencionadas potenciarían el mejoramiento de las escuelas públicas, precisamente por el efecto de competencia (Bellei, 2007). Sin embargo, como señalan Carnoy y McEwan (2003), este argumento nunca se ha dado en la práctica. Asimismo, la OCDE ${ }^{3}$ (2004) afirma que la educación chilena "está influenciada por una ideología que da una importancia indebida a los mecanismos de mercado" (p. 288).

El debate actual de la educación chilena ha sufrido cambios a raíz de las movilizaciones estudiantiles el año 2006. Quizás, el más importante es haber puesto en discusión la pertinencia de la oferta privada subvencionada y cuestionar el lucro que podría hacerse con estos fondos, que eventualmente afectan la calidad de la provisión educativa.

Esta postura ha causado más de una opinión en los actores educativos (Informe del Consejo Asesor Presidencial para la Calidad de la Educación, 2006): por una parte, la derecha política sigue en defensa de la provisión educativa privada con financiamiento del Estado, puesto que de esta forma se aumentaría la cobertura; además, argumenta este sector que la Educación debe permitir la posibilidad de ser con fines de lucro, dado que, "de no haber posibilidad de ganancia, a muchas personas les sería imposible dedicarse a la educación" (p. 18 del tal Informe). Por otra parte, otros actores ligados a la educación pública, al mundo social e incluso a sectores del gobierno de la Concertación ${ }^{4}$ han mostrado su desacuerdo. Se rechaza la existencia de establecimientos educacionales con fines de lucro, dado que la educación es un bien colectivo que no debe ser mercantilizado (Bloque Social, 2006).

La Ley Orgánica Constitucional de Enseñanza ${ }^{5}$ del año 1990, por otra parte, establece que los sostenedores son cooperadores de la función educativa. Sin embargo, de acuerdo a Almonacid (2008), no se especifica en la ley de qué forma se establece dicha cooperación educativa, y sí los requisitos de ingreso y mantenimiento de los sostenedores. El concepto de cooperador de la función educativa adquiere gran relevancia, dado que evidencia la contradicción entre lucro (que es privado, de uno o de unos pocos) y bien común (que refiere al conjunto de la Sociedad). Sería interesante, por tanto, apreciar cómo los entes privados otorgan esta cooperación educativa, por ejemplo estudiando el índice de estudiantes de condición socioeconómica baja en los planteles subvencionados particulares.

Por último, un problema fundamental en términos de transparencia es que no se conoce con exactitud a quiénes el Estado entrega fondos públicos y, menos aún, si éstos son gastados íntegramente en la administración de los establecimientos educacionales

De esta forma, un primer paso importante es conocer a las personas jurídicas y naturales que gestionan más del $40 \%$ de los recursos que el Estado destina a la provisión educativa. Este sector se encuentra compuesto por personas naturales, entidades religiosas u organizaciones filantrópicas, incluye también una importante presencia de sociedades comerciales, de las cuales no se conoce la identidad de quiénes las conforman. Tampoco es posible determinar cuáles son las relaciones que estos actores privados establecen entre sí o con otros sectores productivos. Este punto oscuro en los registros no permite dilucidar con exactitud un sector de la población que 
recibe una gran cantidad de recursos del Estado y que no ha cumplido con los pronósticos de su papel en el sistema educativo.

El objetivo de este artículo es presentar y discutir algunos resultados de la investigación que se ha llevado a cabo para describir y analizar la participación actual de los sostenedores en la provisión educativa particular subvencionada en la Región Metropolitana. Específicamente, en el artículo se busca describir la presencia del sector privado subvencionado en la Región con respecto a otros tipos de administración y caracterizar la oferta educacional particular subvencionada según variables demográficas de dichos establecimientos y variables propias de los sostenedores. Por último, se propone una clasificación de los sostenedores particulares subvencionados y se discuten los resultados.

\section{Antecedentes}

En el caso chileno, la educación privada existe desde los inicios de la República, lo cual no es distinto a lo que ha sucedido en otros países. Vanderberghe (1999) menciona que en los Países Bajos la provisión privada con fondos públicos existe hace un siglo y responde a la exigencia de grupos sociales concretos (las Iglesias) por el derecho a entregar educación. En este sentido, la lucha obedecía a motivaciones ideológicas sobre el rol de Estado vs. Iglesias. Sin embargo, a la revitalización de este debate se suma el rol fundamental atribuido al mercado para que en teoría pudiere éste convertirse en el mecanismo de cobertura y mejora de la calidad de la educación. Tal noción, como se había mencionado, proviene de las ideas esbozadas por Friedman (1956) y desarrolladas posteriormente por Chubb y Moe (1990; en McEwan, 2002), West (1997) entre otros autores.

Claudio Almonacid (1997) señala que intereses comprometidos han favorecido la conformación de un cuasimercado educativo, que se resiste a la transparencia y que utiliza ventajas estructurales que favorecen el crecimiento de la oferta particular subvencionada por las siguientes razones:

Respecto al impacto de la educación privada subvencionada, diversos estudios han argumentado a favor de los establecimientos particulares subvencionados, basándose en la ventaja que éstos obtienen por sobre los colegios municipales en las pruebas nacionales estandarizadas (Aedo y Sapelli, 2001; Gallego, 2002). Sin embargo, el estudio de McEwan y Carnoy (2000) señala que este efecto, en el caso chileno, es producido por los mejores resultados obtenidos por los colegios católicos, quienes elevarían el promedio de los colegios particulares subvencionados. Además, que la evidencia no muestra que la educación chilena haya mejorado con la introducción de la enseñanza privada con financiamiento estatal (Donoso y Hawes, 2002; Carnoy y McEwan, 2003; OCDE, 2004, Bellei, 2007), sino que además, éste es altamente segregado (OCDE, 2004; Redondo et al 2004; Cornejo, 2006; Hsieh y Urquiola, 2006; Bellei, 2007).

Sobre esto último, tomando en consideración el análisis hecho por Almonacid (2004), hay cuatro puntos a considerar:

a) Los resultados en pruebas estandarizadas, cuando son neutralizados por nivel socioeconómico tienden a registrar diferencias muy reducidas o nulas (Mc Ewan y Carnoy, 2003), salvo en el caso de los colegios de administración religiosa. 
Identificación de la Propiedad y Dinámica de la Oferta Educativa Particular Subvencionada de la Región

b) los procesos de selección que emplean esencialmente los colegios privados subvencionados les permiten "descremar" entre estudiantes que rinden académicamente mejor de los que no, y de esta forma exhibir mejores logros ante la sociedad (sobre procesos de selección, ver Parry, 1996)

c) la supuesta efectividad de los establecimientos privados subvencionados, definida como un uso más eficiente de los recursos en función de mejores resultados, oculta diferencias, por ejemplo, en las condiciones laborales de los docentes, sueldos o estabilidad contractual, montos de FICOM y donaciones empresariales.

d) al apreciar los resultados académicos que obtienen los alumnos chilenos en distintas pruebas internacionales (Primer estudio comparativo del Laboratorio de la calidad de la calidad de la Educación, organizado por UNESCO; Tercer Estudio Internacional de Matemáticas y Ciencias, TIMSS) se evidencia que "toda nuestra población, incluidos los sectores de más altos recursos, tienen un rendimiento bajo en relación con los países con buenos niveles educacionales" (Eyzaguirre y Le Foulon, 2001, p. 180). En estas pruebas, Chile ocupa los últimos lugares.

Con respecto al grado de preponderancia que los sostenedores particulares subvencionados poseen en la educación pública, las estadísticas del MINEDUC para el año 2006 señalan que, en cifras, para el año 2006, el sistema educativo chileno registró en la enseñanza parvularia (sin incluir JUNJI ni INTEGRA), básica y media, un total de 11. 671 establecimientos de enseñanza, de los cuales 5.971 son de administración municipal, 4.897 particulares subvencionados, 733 de administración pagada y 70 de administración delegada a corporaciones, empresariales la mayor parte. Este aparente predominio de los establecimientos de administración municipal deja de ser tal cuando se considera que los establecimientos particulares subvencionados por el Estado se concentran en las zonas urbanas, llegándose al punto que en la Región Metropolitana sólo el 27,9 \% del total de establecimientos pertenecen a la educación municipal, en tanto que el 59 \% pertenecen a la educación particular subvencionada (MINEDUC, 2006). Las cifras son aún más drásticas si se considera específicamente la Provincia de Santiago y los establecimientos de enseñanza media.

Sin embargo, más precisamente, se desconoce la identidad y/o grupos de pertenencia de los sostenedores educacionales de la Región Metropolitana. Uno de los escasos antecedentes que se dispone lo representa la investigación de Almonacid (2004) respecto a la identificación de la propiedad de los sostenedores educacionales. Este investigador señala que los sostenedores se pueden clasificar en religiosos (principalmente, adscritos al credo católico, seguido de las iglesias evangélica), sociales (principalmente, clasificados en sostenedores ligados a la beneficencia, fundaciones y empresariales) y los sostenedores privados (desglosados en particulares y en sociedades). Este autor, además, identifica la figura legal de los sostenedores de los establecimientos educacionales nacionales, pero no logra indicar quiénes son los particulares detrás de éstas, como tampoco los nexos eventuales con otros sostenedores, sectores productivos o políticos. Otros antecedentes respecto a la identificación de sostenedores, en el caso específico de los colegios declarados católicos, los presenta Passalacqua (2006) donde señala que un 58\% de los colegios católicos son administrados por religiosos, un $24 \%$ por corporaciones o fundaciones, un $10 \%$ por particulares católicos y un $8 \%$ dependen de directamente de la iglesia católica. 
Actualmente, después de la movilización estudiantil ocurrida el año pasado, es indispensable esclarecer estas interrogantes. Esto es necesario para la elaboración de reformas, sobre todo al sistema de financiamiento de la educación, las cuales tocarán intereses que hoy se encuentran encubiertos en distintos tipos de sociedades y fundaciones que ocultan el real beneficio que ha habido con un negocio que no ha sido realmente transparente.

\section{Metodología}

Para cumplir con los objetivos planteados se llevó a cabo una investigación exploratoria y descriptiva de los sostenedores particular subvencionados de la Región Metropolitana que recibieron subvención el año 2007.

La información utilizada provino de fuentes secundarias, aunque la mayor parte fue resistematizada específicamente para la investigación. Estas son:

- Bases para el año 2007 que el Mineduc publica sobre los establecimientos, en las que se encuentra información sobre el sostenedor o el representante legal y algunas características demográficas de los establecimientos, y también resultados SIMCE y PSU, datos de matrícula y planta docente, entre otros.

- Sistema electrónico del Diario Oficial, en el que se buscó la información sobre cada sostenedor de la muestra: socios de la sociedad, corporación o fundación sostenedora, rut y profesión, participación del o los sostenedores en otras sociedades, los rubros de estas sociedades, sus porcentajes de propiedad, pertenencia a asociaciones gremiales, años de constitución y modificación de las sociedades.

- Buscador en Internet Google, con el cual se buscó completar la información acerca de su pertenencia a grupos políticos, económicos o socioculturales conocidos.

La población objetivo del estudio corresponde a la totalidad de los sostenedores de establecimientos particulares subvencionados por el Estado de la Región Metropolitana que durante el año 2007 percibieron aportes por estudiante matriculado según el DFL2 de 1996 sobre subvención del Estado a establecimientos educacionales $(\mathrm{N}=1355)$. Esta Región fue escogida debido a que en ella se concentra la mayor cantidad de establecimientos particulares subvencionados (1691 en total) en términos absolutos y relativos cuando se compara con el porcentaje de establecimientos de distintas dependencias. Los 1691 establecimientos particular subvencionados de la región son administrados por un total de 1391 sostenedores $\left(\mathrm{N}_{\text {sost. part-subv. }}=1391\right)$.

Cumplir con el objetivo de identificar y caracterizar individualmente a los sostenedores requirió la generación de una nueva base de datos a partir de lo recogido desde el Diario Oficial, para lo cual se estudió una muestra representativa de la población de sostenedores $(n=300)$. Esta muestra tuvo una representación proporcional según tipo de personalidad jurídica. De este modo, se estudiaron 125 sociedades comerciales $(41,7 \%), 136$ personas naturales $(41,7 \%)$ y 39 organizaciones sin fines de lucro, como congregaciones, fundaciones y corporaciones (12,9\%). Este último tipo de sostenedor fue considerado en el análisis general de los establecimientos de la Región Metropolitana, pero no en el análisis que se presenta en este artículo sobre las características de los sostenedores.

\subsection{Variables}


Las fuentes de información consultadas permitieron conocer a los sostenedores en cuanto a las siguientes variables:

- Personalidad jurídica

- Subtipo de personalidad jurídica

- Número de establecimientos sostenidos

- Número de sociedades paralelas a la sociedad o persona sostenedora

- Número de socios y su \% de participación

- Profesión de los sostenedores

- Rubro de las sociedades paralelas

- Año de constitución de la sociedad sostenedora

Se consideró que las características de los establecimientos educacionales también describen a sus sostenedores, por lo que se analizó las siguientes variables:

- Dependencia administrativa

- Comuna establecimiento

- Área geográfica

- FICOM

- Matrícula total

- Índice de Vulnerablidad Escolar ${ }^{6}$ de Enseñanza Básica (IVE EB)

- Índice de Vulnerablidad Escolar de de Enseñanza Media (IVE EM)

- Número de docentes

- Horas/docentes

El carácter exploratorio de le investigación definió una actitud abierta a nuevas variables o combinaciones de ellas que permitieran describir mejor la configuración de la oferta educativa subvencionada, por lo que en el análisis de casos la descripción va más allá de las características descritas.

\subsection{Procedimiento}

El análisis descriptivo de la distribución según ciertas variables del conjunto total de sostenedores se llevó a cabo fundiendo las bases del Mineduc y trabajando una base única con el programa SPSS 11.5.

La recolección de datos desde el Diario Oficial se llevó a cabo con un equipo de estudiantes de la FACSO, que buscó uno a uno la información de los sostenedores de la muestra, sistematizando lo hallado en fichas descriptivas de cada sostenedor. Luego, algunas variables de estas fichas fueron ingresadas a una base Acces, con la cual fue posible cruzar la información de distintos sostenedores, aunque estuvieran invisibilizados en un comienzo por la sociedad que constituían. Posteriormente, se realizó un análisis estadístico vía SPSS 11.5. y un análisis cualitativo. Éste último consistió en revisar la totalidad de las fichas, agrupándolas según una combinación de características compartidas, no definidas a priori. Este trabajo fue realizado en paralelo a la construcción de la base Acces y a su análisis.

\section{Resultados}

3.1. Oferta de provisión educativa en la Región Metropolitana según tipo de administración (año 2007) 
Existe una predominancia del sector particular subvencionado respecto a los otros tipos de dependencia, tanto en lo referente a la cantidad de establecimientos como a la matrícula de alumnos que concentran. Si se observa la siguiente tabla, se advierte que considerando el porcentaje de establecimientos, los particulares casi alcanzan el $60 \%$ del total regional.

Tabla 1 Distribución de los establecimientos y las matrículas de la R.M. según todas las dependencia administrativa

\begin{tabular}{|l|c|c|c|c|}
\hline \multirow{2}{*}{ Dependencia administrativa } & \multicolumn{2}{|c|}{ Establecimientos } & \multicolumn{2}{c|}{ Matrícula } \\
\cline { 2 - 5 } & $\#$ & $\%$ & $\#$ & $\%$ \\
\hline Técnico Profesionales (Decreto Ley 3166) & 33 & $1.19 \%$ & 27.354 & $1.94 \%$ \\
Municipales (Corp. Educ. y DAEM) & 753 & $27.24 \%$ & 444.788 & $31.93 \%$ \\
Particulares Privados: & 325 & $11.76 \%$ & 151.171 & $10.76 \%$ \\
Particulares Subvencionados: & $\mathbf{1 . 6 5 3}$ & $\mathbf{5 9 . 8 0} \%$ & $\mathbf{7 8 1 . 8 8 7}$ & $\mathbf{5 5 . 6 4 \%}$ \\
\hline Total de establecimientos: & 2.764 & $100 \%$ & 2.764 & $100 \%$ \\
\hline
\end{tabular}

En cuanto a la distribución por las 37 comunas de la Región Metropolitana, se destaca que tres de ellas concentran más de un 20 por ciento de los establecimientos administrados por particulares subvencionados. Estas comunas son La Florida (8.6\%), Maipú $(8.0 \%)$ y Puente Alto $(8 \%)$, cuyas características comunes son ser comunas habitacionales y tener una población relativamente reciente, mezcla de clase media emergente con sectores de alta vulnerabilidad social ${ }^{7}$. En contraste a esta concentración, la presencia de sostenedores privados en zonas rurales es mínima, habiendo comunas como Alhué y San Pedro donde es nula.

\subsection{Participación de los distintos tipos de sostenedores particular subvencionados de la Región Metropolitana según zona geográfica, administración, matrícula y número de docentes}

Con respecto a los establecimientos particulares subvencionados, se consideró para el análisis una primera forma de categorización, que se remite a su naturaleza jurídica, diferenciando entre las personas naturales y las personalidades jurídicas, diferenciando entre sociedades comerciales y organizaciones sin fines de lucro, como fundaciones, corporaciones y congregaciones.

Se observa, en este sentido, que son las personas naturales, seguido de las sociedades, las que tienen mayor presencia en la Región Metropolitana, sumando juntas más del 80 por ciento de todos los establecimientos particular subvencionados. Menos de un 20 por ciento del total de sostenedores serían organizaciones sin fines de lucro, como se observa en la siguiente tabla:

Tabla 2 Distribución de Establecimientos Particular Subvencionados de la R.M. según la naturaleza jurídica de su administración

\begin{tabular}{|l|c|c|}
\hline Naturaleza jurídica de la administración & $\#$ & $\%$ \\
\hline Fundaciones, Corporaciones y Congregaciones & 289 & $17.1 \%$ \\
Personas Naturales & 725 & $42.9 \%$ \\
Sociedades comerciales & 677 & $40.4 \%$ \\
\hline Total Establecimientos Privados-Subv. & 1.691 & $100 \%$ \\
\hline
\end{tabular}

Fuente: Mineduc (2007) 
En 1993, año que se aprobó el FICOM, 273 establecimientos particular subvencionados de todo el país optaron por ese sistema. El año 2006, este número había ascendido a 1.963 (Valenzuela, Bellei y De los Ríos, 2008). En la Región Metropolitana la evolución es similar, contándose para el año 2007 un total de 915 establecimientos con Financiamiento Compartido (FICOM), lo que corresponde a un 54,1\% del total regional. Desagregado por tipo de sostenedores particulares subvencionados, se aprecia que son las sociedades comerciales las que más han optado por FICOM, tanto en número absoluto como en porcentaje relativo a su grupo. Las organizaciones sin fines de lucro, por su parte, aunque son el grupo con menor número de establecimientos, destacan por poseer también un mayor número de colegios FICOM (ver tabla 3$)^{8}$.

Tabla 3 Proporción FICOM/ sin FICOM de los establecimientos según tipo de sostenedor

\begin{tabular}{|l|c|c|}
\hline \multirow{2}{*}{ Naturaleza jurídica de la administración } & \multicolumn{2}{|c|}{ Tipo de financiamiento } \\
\cline { 2 - 3 } & Ficom & Sin Ficom \\
\hline Fundaciones, Corporaciones y Congregaciones & $55,0 \%$ & $45,0 \%$ \\
Personas Naturales & $49,7 \%$ & $50,3 \%$ \\
Sociedades comerciales & $58,5 \%$ & $41,5 \%$ \\
\hline Total & $54,1 \%$ & $45,9 \%$ \\
\hline
\end{tabular}

Fuente: Mineduc (2007)

Con respecto a la magnitud de las escuelas, se observa que las corporaciones y fundaciones son las que mayor cantidad de estudiantes concentran, superando en el doble a las personas naturales. En promedio, los establecimientos de corporaciones, fundaciones y congregaciones tienen 679 estudiantes, mientras que los de personas naturales tienen 325 . Las sociedades educacionales, por su parte, tienen un promedio de 584 estudiantes por colegio.

En concordancia con estos datos, existen grandes diferencias respecto a la cantidad de docentes por cada tipo de dependencia. Por ejemplo, en los establecimientos de personas naturales se cuenta sólo con un promedio de 14 profesores por establecimiento; en cambio, en las sociedades educacionales la media de profesores es de 24. Por último, son las organizaciones sin fines de lucro las que mayor cantidad de docentes contratan, con una media de 31 profesores. Asimismo, éstas son las que dedican una mayor cantidad de profesores a otras funciones que no correspondan a la de docente de aula (un promedio de cuatro profesores con funciones distintas, como la técnico-pedagógica y la directiva).

Sin embargo, el promedio de horas de profesor por establecimiento, no difiere significativamente, aun cuando son las sociedades las que concentran mayor cantidad promedio de horas ( 33.5 horas, versus las 32.6 de las personas naturales y las 32.11 de las organizaciones sin fines de lucro).

Respecto a la población que atienden, son las personas naturales las que atienden a los estudiantes más vulnerables dentro del subsector particular subvencionado. Este dato no deja de asombrar, en tanto en el espacio discursivo de la prensa nos encontramos con que las organizaciones sin fines de lucro son las que trabajarían más fuertemente en contextos de pobreza. Los porcentajes promedios del IVE (Índice de Vulnerabilidad Escolar; a mayor IVE, mayor vulnerabilidad) son: 
Tabla 4 Porcentaje Promedio IVE EB y EM según tipo de sostenedor

\begin{tabular}{|l|c|c|}
\hline \multirow{2}{*}{ Naturaleza jurídica de la administración } & \multicolumn{2}{|c|}{ Porcentaje Promedio } \\
\cline { 2 - 3 } & IVE EB & I VE EM \\
\hline Fundaciones, Corporaciones y Congregaciones & $57,04 \%$ & $59,83 \%$ \\
Personas Naturales & $67,57 \%$ & $66,93 \%$ \\
Sociedades comerciales & $60,63 \%$ & $59,37 \%$ \\
\hline
\end{tabular}

Fuente: Mineduc (2007)

\subsection{Caracterización de los sostenedores con posibilidad legal de fines de lucro. ¿Quiénes y cómo son?}

Los resultados que se presentan a continuación describen al conjunto de sostenedores de la Región Metropolitana, no al conjunto de establecimientos (como sucede en el apartado anterior).

La siguiente tabla permite advertir las diferencias:

Tabla 5 Recuento y porcentaje de los establecimientos de la Región Metropolitana y de sus sostenedores, según la naturaleza jurídica de estos últimos

\begin{tabular}{|c|c|c|c|c|c|c|}
\hline \multirow[b]{2}{*}{$\begin{array}{l}\text { Naturaleza jurídica de la } \\
\text { administración }\end{array}$} & \multicolumn{2}{|c|}{$\begin{array}{c}\text { Estableci- } \\
\text { mientos }\end{array}$} & \multicolumn{2}{|c|}{ Sostenedores } & \multicolumn{2}{|c|}{$\begin{array}{c}\text { Sostenedores } \\
\text { muestra }\end{array}$} \\
\hline & \# & $\%$ & \# & $\%$ & \# & $\%$ \\
\hline Fund. Corp. y Congregaciones & 289 & $17.1 \%$ & 179 & $12.9 \%$ & 39 & $12.9 \%$ \\
\hline Personas Naturales & 725 & $42.9 \%$ & 626 & $45.3 \%$ & 136 & $45.3 \%$ \\
\hline Sociedades comerciales & 677 & $40.4 \%$ & 576 & $41.7 \%$ & 125 & $41.7 \%$ \\
\hline Total & 1.691 & $100 \%$ & 1.391 & $100 \%$ & 300 & $100 \%$ \\
\hline
\end{tabular}

Fuente: elaboración propia (datos de 2007)

Para esta etapa del estudio se abordaron las personas naturales y las sociedades comerciales sostenedoras, dejando para otro momento y otro tipo de recolección de datos a las Corporaciones, Fundaciones y Congregaciones, cuya naturaleza jurídica (para el caso de las congregaciones, obediente al derecho canónico) y la serie de leyes que permiten la invisibilidad de sus participantes, impedían el mismo tipo de análisis que los otros tipos jurídicos.

\subsubsection{Frecuencias por distintas clasificaciones de los sostenedores}

- Según la naturaleza jurídica, sin considerar las Corporaciones y Fundaciones Sostenedoras en el total, el $54,5 \%$ de los sostenedores son personas individuales que reciben y administran la subvención por sus establecimientos, mientras que el $46 \%$ son más de una persona.

Tabla 6 Frecuencia y Porcentaje de cantidad de individuos tras la figura de sostenedor

\begin{tabular}{|l|c|c|}
\hline $\begin{array}{l}\text { Número de individuos } \\
\text { directamente sostenedores }\end{array}$ & $\#$ & $\%$ \\
\hline $1 *$ & 142 & $54,5 \%$ \\
2 & 51 & $19,7 \%$ \\
3 & 28 & $10,8 \%$ \\
4 & 28 & $10,8 \%$ \\
5 & 6 & $2,4 \%$ \\
6 o más & 6 & $2,4 \%$ \\
P. nat. y Soc. comerciales & 261 & $100 \%$ \\
\hline \multicolumn{2}{|c|}{ *Caso de Personas Naturales y Empresas Individuales }
\end{tabular}

Revista Mad. N²0, Mayo de 2009. Departamento de Antropología. Universidad de Chile http://www. revistamad.uchile.cl/20/corbalan_05.pdf 
de responsabilidad limitada (E.1
Fuente: elaboración propia (datos de 2007 )

Tomando sólo a las sociedades comerciales sostenedoras, el $41 \%$ de éstas está constituida por sólo dos personas, cuya proporción de participación varía entre 50:50 y 99: 1. El $45 \%$ son 3 o 4 personas en la sociedad. Hay algunas pocas sociedades donde hay hasta 50 participantes, los que típicamente son profesores asociados. Sin embargo, en muchos de esos casos, los profesores delegan la administración del establecimiento en el antiguo dueño, en alguno de los socios o en la Corporación municipal ${ }^{9}$.

Las sociedades comerciales se subclasifican en sociedades de responsabilidad limitada (S. Ltda., 81,8\%), sociedades anónimas (S.A, 18,9\%). y empresas individuales de responsabilidad limitada (E.I.R.L., 3,8\%).

Tabla 7 Frecuencia y Porcentaje de tipos de sociedades comerciales

\begin{tabular}{|l|c|c|}
\hline $\begin{array}{l}\text { Tipos de sociedades } \\
\text { comerciales }\end{array}$ & $\#$ & $\%$ \\
\hline S. Ltda. & 102 & $81,8 \%$ \\
S.A. & 19 & $18,9 \%$ \\
E.I.R.L & 4 & $2,9 \%$ \\
Soc. comerciales & 125 & $100 \%$ \\
\hline
\end{tabular}

Fuente: elaboración propia (datos de 2007)

- Según los rubros o giros sociales establecidos, la gran mayoría de las sociedades sostenedoras define específicamente la administración de establecimientos educativos (de distinto tipo), superando el $95 \%$ del total ${ }^{10}$.

- Según la relación familiar de los socios, de las sociedades comerciales que pudo conocerse los datos, el $43.4 \%$ está constituida por personas que no poseen, aparentemente, relación familiar alguna. El $11.8 \%$ de las sociedades comerciales tienen algunos de sus socios claramente relacionados familiarmente y el $44.7 \%$ tienen a todos sus socios relacionados familiarmente, destacándose, típicamente, la configuración Esposo y/o Esposa e Hijos.

- Según los años de constitución de las sociedades sostenedoras, se observó más de la mitad de las sociedades que actualmente administran establecimientos fueron constituidas hace menos de 8 años. En detalle, el 5.1\% fueron creadas antes de 1985, el $11.5 \%$ entre 1985 y 1989, el $9 \%$ entre 1990 y 1994, 23.1\% entre 1995 y 1999 y el $51.3 \%$ a partir del año 2000. No se conoce los años en que las Personas Naturales comenzaron a recibir subvenciones del Estado.

Tabla 8 Frecuencia y porcentaje de las sociedades comerciales por periodos de constitución

\begin{tabular}{|l|c|c|}
\hline Periodos de constitución & $\#$ & $\%$ \\
\hline Sin dato & 20 & $16,1 \%$ \\
Antes de 1985 & 6 & $4,0 \%$ \\
$1985-1989$ & 12 & $9,6 \%$ \\
$1990-1995$ & 9 & $7,5 \%$ \\
$1995-1999$ & 24 & $1,9 \%$
\end{tabular}




\begin{tabular}{|l|c|c|} 
Después de 2000 & 54 & $43,0 \%$ \\
Total sociedades comerciales & 125 & $100 \%$ \\
\hline \multicolumn{2}{|c|}{ Fuente: elaboración propia (datos de 2007) }
\end{tabular}

\subsubsection{Número de establecimientos educacionales por sostenedores}

El $87 \%$ de los sostenedores, individuales o asociados, administra sólo un establecimiento, mientras que el $9.4 \%$ administra 2. El resto administra desde 3 hasta casos muy excepcionales de hasta 17 establecimientos (aunque en la muestra el máximo es 5).

Se observó que no hay diferencias significativas de cantidad de establecimientos según se trate de una Persona Natural o una Sociedad Sostenedora.

Existen casos excepcionales de Personas Naturales Sostenedoras ( $4 \%$ de la muestra) que actualmente también participan de sociedades que sostienen otros establecimientos educacionales. Esto significa que el recuento de establecimientos no es preciso si se considera sólo la figura directa del sostenedor y no su composición. En este sentido, está pendiente el recuento de los establecimientos que los socios de sociedades sostenedoras poseen, tomando en cuenta las distintas sociedades en las que participa.

\subsubsection{Profesiones de los sostenedores}

Las profesiones fueron conocidas para la gran mayoría de las personas que en el Diario Oficial aparecen participando de alguna sociedad y/o asociación y/o sujetos a alguna Prenda. Por este motivo, sólo para aquellas personas naturales sostenedoras presentes en el Diario Oficial, fue posible tener este dato ( $30 \%$ del total de personas naturales). Considerando que aparecer o no en el Diario Oficial no depende de la profesión, vale tomar en cuenta las proporciones encontradas, que refieren que el $89.6 \%$ de las Personas Naturales Sostenedoras son educadoras (profesor, psicopedagogo, parvulario), el $6.3 \%$ ejerce una profesión ligada con la Administración y el comercio, el $2.1 \%$ una profesión ligada a la salud y un $2.1 \%$ ligado a ingenierías o técnicas.

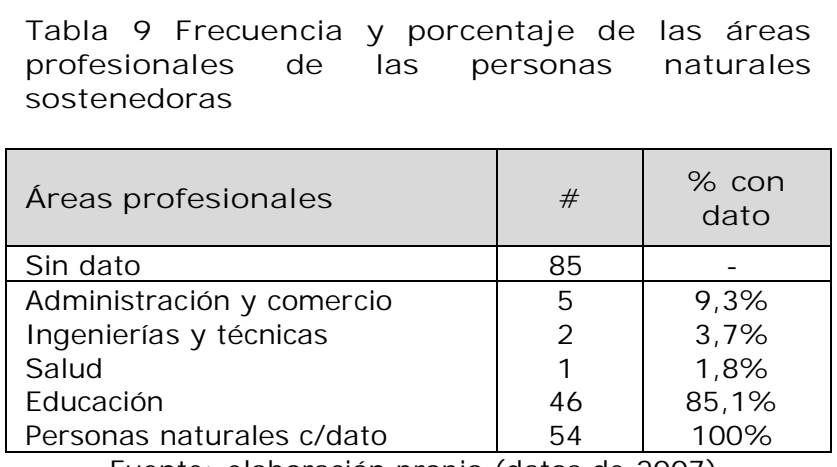

Fuente: elaboración propia (datos de 2007)

Por otro lado, se observó que de las sociedades sostenedoras, sólo el $71.3 \%$ de ellas posee al menos a un educador entre sus miembros. 


\subsubsection{Sociedades de los sostenedores}

El $46.5 \%$ de las personas naturales Sostenedoras participa en sociedades comerciales, destacándose entre ellas las educacionales, las de comercio de productos, las empresas de servicio y las inmobiliarias. Por otra parte, el $55 \%$ de los socios de sociedades sostenedoras participa en otras sociedades, destacándose los mismos rubros que para las personas naturales, pero añadiéndose el rubro de Transportes y Telecomunicaciones y el de Servicios Financieros. Una diferencia que existe entre uno y otro grupo es que por un lado, muy pocas personas naturales participan en más de tres sociedades comerciales (sólo el $5 \%$ ), mientras que los socios de sociedades sostenedoras participan frecuentemente en 4, 5 y hasta 17 sociedades (casi el $30 \%$ ).

\subsubsection{Clasificación de los sostenedores particular-subvencionados según las características revisadas}

Las únicas clasificaciones que significan un trato distinto de parte del MINEDUC hacia los sostenedores repercuten sobre la entrega de recursos, las exigencias sobre selección y las exigencias con respecto al Estatuto Docente. Para la entrega de recursos por concepto de subvención, a excepción de los establecimientos de administración delegada a Corporaciones empresariales, existe igualdad de trato hacia municipios o privados. Así, la diferencia en montos de subvención no depende de la naturaleza de la contraparte, sino de las características de los establecimientos: zona geográfica, tipo de estudiantes que atiende (alumnos con necesidades especiales, vulnerables e indígenas tienen otros montos asociados), nivel y modalidad de enseñanza, proyectos de infraestructura o de mejoramiento institucional. Estas variables son las primeras que hacen diferencias entre todos los sostenedores.

Esta investigación revela características diferenciadoras que hasta el momento no son consideradas en la relación con el Ministerio o con las familias de estudiantes, como su personalidad jurídica (S.A./S.Ltda./E.I.R.L/Persona Natural/Corporación/ Fundación/Congregación); su finalidad (lucro/no lucro); su modelo de desarrollar el negocio (nr.; tipo; comunas de establecimientos); el nivel de complejidad de su propiedad (una persona, varias, sociedades, agrupaciones); el derecho bajo el que se rige (civil/canónico); los intereses societales/comerciales de los sostenedores

A partir de estos criterios, el análisis cualitativo de los tipos de sostenedores permite proponer la siguiente clasificación tentativa de tipos de sostenedores:

1) "Los asalariados": Son sostenedores que suelen tener un solo establecimiento, con pocos estudiantes comparativamente. Son personas naturales o sociedades pequeñas, de baja inversión. Muchos son profesores que solos o asociados con algún familiar administran su propio establecimiento, siendo generalmente sus directores. Estas personas o los socios de estas sociedades sostenedoras generalmente no tienen otros negocios paralelos.

2) "Los pequeños comerciantes": Son sostenedores que también suelen tener un establecimiento 0 a lo sumo dos. Como personas naturales o sociedades educacionales, suelen estar ligados a otras empresas de rubro asociado a los establecimientos educacionales, como empresas de transporte, confección de vestimenta, venta de artículos escolares, venta de alimentos, etc. Estas otras empresas son administradas por ellos mismos y algún familiar, que en muchas 
ocasiones tiene por su cuenta otras sociedades que puede suponerse ligadas al eje del establecimiento. En la mayoría de los casos la persona natural o el accionista mayor tiene una profesión ligada a la educación. Algunos de estos sostenedores tienen una configuración invisibilizante de su composición: en los casos simples, tras sociedades anónimas; en los casos más complejos, combinan en sus sociedades personas naturales de bajo aporte a la sociedad con sociedades de mayor aporte, que a su vez están constituidas por las mismas personas naturales (esto en varias capas).

3) "Los inversionistas": Son sostenedores que se organizan en sociedades compuestas por otras sociedades (como holdings). Los giros sociales son educacionales, pero las sociedades que las componen suelen ser inmobiliarias, constructoras o financieras. Los montos iniciales son cuantitativamente mucho más altos que los de las sociedades antes descritas, superando en varios casos los 100 millones de pesos. Estos sostenedores poseen dos o más establecimientos, marcando la diferencia en la matrícula que atienden (siempre más de 500 alumnos). Las personas que están tras estas grandes sociedades de sociedades, generalmente no son docentes. El análisis cronológico de las sociedades de sus socios permite hacer una subclasificación: a) los que iniciaron la carrera comercial desde un rubro educacional, añadiendo con el tiempo sociedades de otros rubros, b) los que constituyeron sociedades educacionales con altos montos de inversión a partir de sociedades financieras o inmobiliarias poderosas.

4) "Los ideológicos": Son sostenedores que se organizan en sociedades sin fines de lucro, es decir, corporaciones o fundaciones. Esto sugiere que sus intereses no son comerciales, sino ideológicos. Se subclasifican en religiosos, laicos y laicos ligados a grupos religiosos. Algunos de estos sostenedores tienen más de 15 establecimientos, con un promedio de matrícula sobre los 600 alumnos. Este grupo también es el que consistentemente aparece más en el espacio discursivo de la prensa y congrega dentro de sí a los principales grupos de influencia en la sociedad civil, como la Iglesia Católica, los grandes empresarios y políticos y ex funcionarios de gobierno, los cuales en su mayoría aboga por la defensa de la Educación privada subvencionada por el Estado esencialmente de la misma forma que se viene realizando ${ }^{11}$.

\section{Discusión}

Con las dificultades antes señaladas respecto a la recolección de información por parte de los sostenedores que administran $\mathrm{y} / \mathrm{o}$ son propietarios de establecimientos particulares subvencionados, el presente artículo da cuenta de las distinciones respecto a cómo se configuran los actores detrás de la oferta privada financiada por el Estado. Sin embargo, la complejidad de la información dificulta realizar un análisis acabado de las redes en relación al grado de concentración o de imbricación de los sostenedores en la Región Metropolitana, lo cual constituye un desafío a enfrentar en el futuro.

Un primer punto importante que surge al apreciar el panorama de la educación privada con fondos públicos es que en Chile es la masificación de la escuela particular subvencionada. Para el caso, viene mencionar dos ejemplos: uno de ellos es la distribución territorial, en donde se aprecia la extensión por sobre un 80 por ciento del total en comunas donde hay un componente importante de la clase media o de segmentación profunda intracomunal: tales serían los casos antes señalados de La Florida, Maipú y Puente Alto; sin embargo, la oferta privada es inexistente en comunas pobres y de pocos habitantes; por otra parte, la participación de personas no 
vinculadas primariamente a la Educación (por ejemplo, ingenieros comerciales, abogados) en diversas sociedades tanto educacionales como en negocios asociados revela un sistema altamente eficaz en captar el ingreso de inversores.

Los datos respectivos también generan interrogantes respecto al poder relativo de los distintos sostenedores. Si bien es cierto que las personas naturales son la modalidad más común para sostener establecimientos, es igualmente cierto que atienden en promedio la mitad menos de estudiantes que las sociedades y organizaciones sin fines de lucro. Pero lo más importante es que su índice de vulnerabilidad escolar es mucho mayor que las de las otras formas de administración. Esto es sorprendente sobre todo si se considera el caso de muchas fundaciones y corporaciones que publicitan que su trabajo está asociado a la población más desfavorecida; sin embargo, en la práctica se aprecia que la proporción de estudiantes vulnerables no las diferencia de lo que realizan otros sostenedores no asociados a la filantropía.

Por otra parte, parece claro que las distinciones respecto a los sostenedores deberían apuntar cada vez más a una mayor precisión. El sector particular subvencionado exhibe una heterogeneidad que no ha sido aprehendida por las categorías tradicionales, lo cual constituyó uno de los objetivos del presente artículo, es decir, apreciar la complejidad de la configuración del mercado educativo en cuanto a sus actores oferentes. Una mayor precisión de los actores nos permite conocer también sus demandas y las consecuencias que tendrían para este subsistema educativo cualquier política tomada por el Estado.

Los tipos de sostenedores identificados y descritos posibilitan entender en alguna medida la respuesta de algunos sectores ante las movilizaciones de estudiantes del 2006, puesto que éstas anunciaban vientos de cambio. La estrategia del lucro apuntó los dardos hacia aquellas sociedades que mezclaban el rubro educacional con otras empresas, dejando inmune los grandes holdings empresariales que tienen entre uno de sus múltiples sociedades una dedicada a la educación y a las corporaciones y fundaciones, cuyos fines no comerciales los muestra aparentemente como volcados al espacio público.

La exploración de las variantes de los proveedores privados de educación y la identificación de distintos tipos de sostenedores revela que, al igual que en el caso comparativo municipales/privados, la igualdad de trato en entrega de subvención no es adecuada si se desea mejorar el servicio educativo que se pretende entreguen las unidades educativas. Con esto se alude a los negocios que algunos poseen mucho más recursos, ya sea desde sus otros negocios o por donaciones "ideológicas", lo que le permite hacer más atractiva sus ofertas y de paso superar ampliamente el número de matriculados, haciendo más eficiente el uso de recursos de la subvención. Como resultado, mientras algunos sectores clave son educados por un grupo pequeño de sostenedores, provenientes de los sectores dominantes, de dudoso interés por el desarrollo integral -y no industrial- de la ciudadanía general, por otro lado, gran parte de la población regional recibe el servicio educativo de privados de pocos recursos, que recurren a congelar las mejoras en las condiciones laborales docentes y a otras estrategias para mantener una matrícula suficiente y así, sobrevivir como empresa.

Uno de los desafíos que queda pendiente a partir de esta investigación es la mayor precisión y operacionalización de la clasificación tentativa propuesta. En este sentido, un paso a dar es establecer la magnitud de cada uno de los cuatro tipos de sostenedores del sector particular subvencionado chileno. Por otra parte, también 
surge como necesidad explorar el marco ético-normativo presente en cada uno de los tipos de sostenedores, expresados, por ejemplo, en su Proyecto Educativo Institucional y los Reglamentos de Convivencia. Es posible que dicha exploración complejice las características de la tipología antes enunciadas y posibilite un acercamiento más acabado hacia los alcances de la provisión educativa subvencionada particular. RM

\section{Bibliografía}

1. Aedo, C. y Sapelli, C. (2001). El Sistema de Vouchers en la Educación: una Revisión de la Teoría y Evidencia Empírica para Chile. Estudios Públicos. 81.

2. Almonacid, C. (1997). El Empresario Chileno como Actor Educativo. Tesis (Doctor en Ciencias de la Educación). Pontificia Universidad Católica de Chile.

3. Almonacid, C. (2004). Un Cuasimercado Educacional: La Escuela Privada Subvencionada en Chile. Revista de Educación, 333. 165-196

4. Almonacid, C. (2008). La Educación Particular Subvencionada como Cooperadora del Estado. En Bellei, C., D. Contreras y J. P. Valenzuela: La Agenda Pendiente en Educación. Profesores, Administradores y Recursos: Propuestas para la Nueva Arquitectura de la Educación Chilena. Programa de Investigación en Educación Universidad de Chile / UNICEF

5. Bellei, C. (2007). Expansión De La Educación Privada Y Mejoramiento De La Educación En Chile. Evaluación A Partir De La Evidencia. Revista Pensamiento Educativo. 17(1).

6. Bloque Social de Educación (2006). Propuestas del Bloque Social a las Organizaciones Populares y a la Ciudadanía para Mejorar la Educación Chilena. Extraído de http://www.opech.cl/bibliografico/doc_movest/documento_propuestas_bloque.pd f el 12 de noviembre de 2007

7. Carnoy, M y McEwan, P. (2003). Does Privatization Improve the Education? The Case of Chile's National Voucher Plan." In Choosing Choice: School Choice in International Perspective. Ed. David N. Plank and Gary Sykes. New York: Teachers College Press

8. Constitución Política de Chile. (1980) Dto. №.1150, 1980.

9. Cornejo, R. (2006). El Experimento Chileno en Educación 20 Años Después: Una Mirada Crítica a los Logros y Falencias del Sistema Escolar. Revista Electrónica Iberoamericana sobre Calidad, Eficacia y Cambio en Educación (REICE), 4 (1). 118-129

10. Donoso, S. (2006). Financiamiento de la Educación Pública en los '90. Cadernos de Pesquisa. 36 (127). 151-172

11. Donoso, S. y G. Hawes (2002). Eficiencia Escolar y Diferencias Socioeconómicas: A propósito de los resultados de las pruebas de medición de la calidad de la educación en Chile. Educação e Pesquisa. 28 (2). 25-39 
12. Eyzaguirre, B. y C. Le Foulon, C. (2001). La Calidad de la Educación Chilena en Cifras. Estudios Públicos, 84. 85-204

13. Friedman, M. (1956). The Role of Government in Education. Versión reimpresa extraída http://www.eco.utexas. edu/facstaff/Cleaver/350kPEEFriedmanRoleOfGovttable.p df el 7 de noviembre de 2007

14. Gallego, F. (2002). Competencia y Resultados Educativos. Teoría y Evidencia para Chile. Cuadernos de Economía. 39 (118), pp. 309-352

15. Hsieh, Ch. T., y M. Urquiola (2006). The Effects of Generalized School Choice on Achievement and stratification: Evidence from Chile's Voucher Program. Journal of Public Economics, 90. 1477- 1503

16. Informe del Consejo Asesor Presidencial para la Calidad de la Educación (2006) Extraído de Extraído de www.consejoeducacion.cl/articulos/Informefinal.pdf el 10 de noviembre de 2007

17. McEwan, P. J. (2002). Public Subsidies for Private Schooling: a Comparative Analysis of Argentina and Chile. Journal of Comparative Policy Analysis: Research and Practice. 4. 189-216

18. McEwan, P. J. y Carnoy, M. (2000). The Effectiveness and Efficiency of Private School in Chile's Voucher System. Educational Evaluation And Policy Analysis, 22 (3). 213-239.

19. MIDEPLAN (2007). Base de Datos de la Encuesta de Caracterización Socioeconómica 2006, CASEN 2006. Extraído de www.mideplan.cl

20. MINEDUC (2006). Anuario Estadístico 2006 Provisorio. Departamento de Estudios y Desarrollo, Ministerio de Educación, actualizado al 30 de junio del año señalado. Extraído de http://w3app.MINEDUC.cl/DedPublico/anuarios_estadisticos el 24 de Octubre de 2007

21. Nef, J. (1999-2000). El Concepto de Estado Subsidiario y la Educación como Bien de Mercado: un Bosquejo de Análisis Político. Revista Enfoques Educacionales. 2 (2), pp. 11-16.

22. OCDE (2004). Revisión de Políticas Nacionales de Educación: Chile. Paris: OCDE.

23. Parry, T. R. (1996). Will Pursuit of Higher Quality Sacrifice Equal Opportunities in Education? An Analysis of the Education Voucher System in Santiago. Social Science Quarterly. 77 (4), pp. 821-841.

24. Passalacqua, A. (2006). ¿Cuántos Somos? ¿Cómo lo Hacemos? Estadísticas Educación Católica Chilena. Primer Congreso Educación Católica 18,19,20 de Octubre 
25. Redondo, J., K. Rojas, y C. Descouvières (2004). Equidad y Calidad en la Educación Chilena. Reflexiones e Investigaciones de Eficiencia de la Educación Chilena Obligatoria. Santiago de Chile: Universidad de Chile. Vice-Rectoría de Investigación, Facultad de Ciencias Sociales.

\section{Valenzuela, J. P., C. Bellei y D. de los Ríos. (2008). Evolución de la Segregación Socioeconómica de los Estudiantes Chilenos y su Relación con el Financiamiento Compartido.}

\section{Vanderberghe, V. (1999). Combining Market and Bureocratic Control in Education: an Answer to Market and Bureocratic Failure? Comparative Education. 35 (3), pp. 271-282}

\section{West, E. G. (1997). Education Voucher in Principle and Practice. World Bank Research Observer. 12 (1). 83-103}

\section{Notas}

1 A partir de la Reforma, se definen 4 tipos de establecimientos subvencionados, según su tipo de administración: 1) Corporaciones Municipales, 2) Departamentos Municipales, 3) Estatales de administración delegada a Corporaciones Empresariales, 4) Privados (Ilamados "particular subvencionados"). El quinto tipo de establecimientos no es subvencionado y se le denomina Particular Pagado.

2 Coalición de gobierno que se identifica con la centro-izquierda, en ejercicio desde el año 1990.

${ }^{3}$ La OCDE es la Organización para la Cooperación y el Desarrollo Económicos, agrupa a 30 países miembros comprometidos a promover la democracia y el libre mercado. Entre sus objetivos, según el artículo 1 de la Convención OCDE, se encuentran: apoyar el crecimiento económico; aumentar el empleo; mejorar la calidad de vida; mantener la estabilidad financiera; asistir a otros países para alcanzar el desarrollo económico y contribuir al crecimiento del comercio mundial. Constantemente realizan estudios respecto a distintas temáticas como Educación y en distintos países. Curiosamente, siendo éste un organismo del capitalismo mundial, realiza una "Revisión de Políticas Nacionales de Educación" cuya evaluación es más bien negativa respecto del clivaje Mercado-Educación introducido por Chile desde la década de 1980. Más información, www.oecd.org

${ }^{4}$ Cabe hacer notar, sin embargo, que el modelo de Educación basada en mecanismos de mercado se ha perfeccionado durante este período, si bien es cierto hay algunos elementos de reversión, pero que no han tocado los pilares fundamentales de lo que podría Ilamarse la "Escuela Chilena Mercantilizada"

${ }^{5}$ Ley promulgada pocas horas antes del término de la dictadura militar que gobernó a Chile desde 1973. Esta ley estableció diversos pilares que resguardaron en lo esencial el modelo de Educación que se había implantado durante este régimen. A la fecha de la redacción del presente artículo, esta ley está a punto de ser reemplazada por una Ley General de Educación, la cual no toca los pilares fundamentales del modelo de Educación chilena, y sí se enfoca en una tecnificación de la escuela subvencionada (municipal y particular) en aras de una mejora de la "calidad"

${ }^{6}$ Indicador construido por la Junta Nacional de Auxilio Escolar y Becas (Junaeb) que estima el porcentaje de la matrícula del establecimiento educacional en el que un estudiante cursó la Enseñanza Básica que se encuentra en condición de vulnerabilidad (considera escolaridad y ocupación de los padres, además de otras variables socioeconómicas).

${ }^{7}$ Ver encuesta CASEN 2006 (Mideplan, 2007)

${ }^{8}$ Un dato complementario es que el $83 \%$ de los colegios católicos recibe subvención del Estado (contando entre ellos a los dependientes de congregaciones, del Obispado y de particulares reconocidos por el Obispo). Si entre estos establecimientos subvencionados católicos, el año 1998 el 39\% tenía FICOM, el año 2002 este porcentaje había subido a un 45\% (Passalacqua, 2006).

${ }^{9}$ Un ejemplo de posesión de los profesores de la sociedad, pero sin ser ninguno de ellos representantes legales, es el Liceo Rafael Sotomayor de Las Condes, administrado por la Corporación de dicha Municipalidad.

${ }^{10}$ La nuevo Ley General de Educación obliga a que las sociedades sostenedoras definan como giro único fines educativos.

11. Como ejemplo de este grupo, se cuenta la Corporación Aprender, que congrega a grandes empresarios como financistas y a dos ex ministros de Educación: Mariana Aylwin y Sergio Molina 